\title{
Whole-body vibration decreases delayed onset muscle soreness following eccentric exercise in elite hockey players: a randomised controlled trial
}

\author{
Harold Akehurst ${ }^{1}$, John E. Grice ${ }^{1,2}$, Manuela Angioi ${ }^{2}$, Dylan Morrissey ${ }^{2}$, Filippo Migliorini ${ }^{3^{*}}$ (ID and
}

Nicola Maffulli ${ }^{2,4}$

\begin{abstract}
Background: Delayed onset muscle soreness (DOMS) is a common non-structural muscle injury which can disrupt training and impair performance in elite athletes. Vibration therapy reduces inflammation and improves neuromuscular efficiency, leading to reductions in pain and stiffness, and may be effective for the prevention or treatment of DOMS. However, the effect of whole-body vibration (WBV) used after sport in elite athletes has not been reported.

Methods: A randomised, controlled trial was performed. Participants were elite (national or international level) hockey players and underwent an eccentric exercise protocol previously shown to produce clinical DOMS. After exercise, one group underwent static stretching with WBV therapy, and the other performed stretching only. Baseline and serial post-exercise pain scores and measurements of quadriceps tightness were obtained.

Results: Eleven participants were recruited into each study arm. There were no significant differences in baseline group characteristics. Participants receiving WBV had significant reductions in both pain $(p=0.04)$ and quadriceps tightness ( $p=0.02$ ) compared with stretching only.

Conclusions: Post-exercise WBV is effective in elite hockey players to reduce DOMS after eccentric exercise. Elite athletes in multi-sprint sports are at risk of DOMS during training and competition, and its reduction could contribute to reduced injury risk and improved performance. This treatment modality is favourable because it can be incorporated with minimal disruption into the recovery section of existing training regimes. These findings may also be extrapolated to other multi-sprint sports.
\end{abstract}

Keywords: Delayed onset muscle soreness, DOMS, Whole-body vibration, WBV, Elite athletes, Eccentric exercise

\section{Background}

Delayed onset muscle soreness (DOMS) was described in 1900 by Hough, who reported soreness and loss of function developing around $12 \mathrm{~h}$ after exercise and lasting

*Correspondence: migliorini.md@gmail.com

${ }^{3}$ Department of Orthopaedic, Trauma, and Reconstructive Surgery, RWTH Aachen University Hospital, Pauwelsstraße 30, 52074 Aachen, Germany

Full list of author information is available at the end of the article several days, which he attributed to muscle fibre damage and inflammation [1]. This description remains remarkably consistent with current understanding, which adds a number of theories for the specific mechanisms of DOMS, including lactic acid, muscle spasm, connective tissue damage, muscle damage, inflammation and enzyme efflux [2-5]. Non-structural injuries such as DOMS account for $70 \%$ of muscle injuries in soccer players, and more than $50 \%$ of days absent from sport 
activity and training [6, 7]. Significant DOMS persists for at least $72 \mathrm{~h}$ following professional soccer matches, resulting in increased training injury risk and impacting on performance [8]. DOMS is more severe in untrained individuals or following an unaccustomed activity in athletes and may result from excessive and prolonged eccentric muscle contractions $[2,9]$. Its prevention and treatment therefore have significant implications both for performance at the elite level and for sustaining participation at the recreational level. A number of methods of prevention or treatment of DOMS have been investigated, including massage [10], cryotherapy [11], active recovery [12], homeopathy [13], acupuncture [14], TEN [15], ultrasound [16], non-steroidal anti-inflammatories [17], steroids [18], vitamin C and antioxidants [19], but consensus support for any single method remains to be established [2] (Table 1). Table 2 provides serial measurements of pain and tightness abrasion therapy which has been proposed in a number of applications in sport. The acute effects of vibration include reduced inflammation, reduced pain, increased flexibility, increased

Table 1 Baseline group characteristics

\begin{tabular}{lll}
\hline Endpoints & WBV & Stretch only \\
\hline Sex & & \\
Male & $7(64 \%)$ & $7(64 \%)$ \\
Female & $4(36 \%)$ & $4(36 \%)$ \\
Age (years) & 26 & 27 \\
Competition level & & $5(45 \%)$ \\
International & $6(55 \%)$ & $6(55 \%)$ \\
National & $5(45 \%)$ & $1(9 \%)$ \\
Usual training (hours/week) & & $9(82 \%)$ \\
$2-5$ & $0(0 \%)$ & $1(9 \%)$ \\
$5-10$ & $9(82 \%)$ & $10(91 \%)$ \\
$>10$ & $2(18 \%)$ & $1(9 \%)$ \\
Body mass index $\left(\mathrm{kg} / \mathrm{m}^{2}\right)$ & $11(100 \%)$ & \\
$20-25$ & 0 & \\
$>25$ & &
\end{tabular}

neuromuscular efficiency and increased strength [2025]. Vibration therapies may be broadly categorised by whether they are applied locally or to the whole body (WBV), and whether they are used before, during or after exercise; specific characteristics including the frequency of vibration and the number and duration of applications vary widely. Vibration therapy has been studied extensively in the specific context of DOMS [26]. It has been proposed that vibration may optimise muscle performance by synchronising motor unit activity and increasing blood flow and that this may prevent mechanical sarcoma disruption and in turn reduce DOMS [26-28]. The overall picture is encouraging, with meta-analysis finding vibration effective for prevention or treatment of DOMS $[29,30]$. Therapies studied include those provided before [31-36] and after [9, 20, 35, 37-49] exercise; in athletes [36, 49], untrained [9, 31-35, 37, 38, 40, 42, 45, $47,48]$ and recreationally active $[20,39,41,43,44,46]$ participants; and using local vibration $[20,31,32,34,35$, $37,38,40,45,48,49]$ and WBV [9, 33, 36, 39, 41-44, 46, 47]. However, the specific case of WBV used post-exercise in elite athletes has not previously been examined. This may represent a significant knowledge gap, as the development of DOMS does vary with level of training, and corresponding variation may be anticipated in therapeutic effects of WBV [50, 51]. Hockey is a multi-sprint sport with similar movements to soccer, yet additionally has multiple repetitions of eccentric exercises such as lunge tackling. Field hockey players are particularly at risk of DOMS, which can harm performance [52]. Elite hockey players competing in sequential games in a tournament are less able to sprint in the second and third games, with DOMS being a probable contributing factor [52].

The primary aim of this study was to assess whether WBV-administered post-eccentric exercise is more effective to reduce DOMS in elite hockey players than static stretching. The objectives were to produce clinical DOMS using a validated exercise protocol, to obtain

Table 2 Serial measurements of pain and tightness

\begin{tabular}{|c|c|c|c|c|c|c|c|}
\hline \multirow[t]{2}{*}{ Endpoints } & \multirow[t]{2}{*}{ Baseline } & \multicolumn{5}{|l|}{ Follow-up } & \multirow[t]{2}{*}{$P$ value ${ }^{\dagger}$} \\
\hline & & Day 0 & Day 1 & Day 2 & Day 3 & Day 7 & \\
\hline \multicolumn{8}{|l|}{ Pain (VAS) } \\
\hline Stretch only & $0 \pm 0$ & $27.2 \pm 20.0$ & $29.80 \pm 18.2$ & $25.3 \pm 23.2$ & $17.5 \pm 23.2$ & $1.3 \pm 4.2$ & 0.04 \\
\hline WBV & $0 \pm 0$ & $32.1 \pm 21.1$ & $27.6 \pm 19.1$ & $11.2 \pm 11.6$ & $2.6 \pm 5.1$ & $0 \pm 0$ & \\
\hline \multicolumn{8}{|c|}{ Tightness (cm) } \\
\hline Stretch only & $12.5 \pm 4.2$ & $13.6 \pm 4.0$ & $15.0 \pm 4.0$ & $15.3 \pm 4.9$ & $15.0 \pm 4.8$ & $12.9 \pm 4.3$ & 0.02 \\
\hline WBV & $13.8 \pm 3.5$ & $14.1 \pm 4.3$ & $14.7 \pm 3.7$ & $14.5 \pm 3.4$ & $13.6 \pm 2.9$ & $12.4 \pm 3.3$ & \\
\hline
\end{tabular}

${ }^{\dagger}$ For difference in area under the curve 
serial pain and functional measurements, and to compare these between treatment groups.

\section{Materials and methods}

\section{Ethics}

Approval was obtained from the Queen Mary Research Ethics Committee (QMREC2012/50). Participants were provided written and verbal information about participation. All subjects gave informed consent to participate.

\section{Population, randomisation and sampling}

Elite (National league or International) field hockey players above the age of 18 were invited to volunteer for the study. Both male and female hockey players were invited. Exclusion criteria were pre-existing injury, systemic disease, muscle disease or previous surgery to the lower limb and non-provision of consent.

Using a closed envelope method, participants were randomly allocated to the intervention or control group. Randomisation, enrolment and assignment were performed by one of the investigators (JG). All players were prescribed a standard eccentric exercise protocol [9] and underwent the same testing procedure at $24,48,72 \mathrm{~h}$ and at 1 week. Only the intervention group underwent the WBV protocol.

Other active recovery work was prevented alongside massage and cryotherapy. Additionally, the use of compression clothing or anti-inflammatories was forbidden in the post-exercise period to avoid bias. Consequently, the hockey off-season period was identified as an appropriate period for testing. Data were collected at Nuffield Health Gym, Surbiton, UK, and Fareham Leisure Centre, Fareham, UK.

\section{Sample size}

Sample size was calculated based on a primary outcome of difference between areas under the curve (AUC) of serial pain visual analogue score (VAS) from day 0 to day 7 post-exercise, anticipating a difference of 5 points per day corresponding with a difference in AUC of 40 pointdays. Based on a standard deviation for the AUC (derived from the reported standard deviations in pain VAS following a comparable exercise regime) [9], two equal groups of $n=8$ were calculated to provide $90 \%$ power at the 0.05 level of significance.

\section{Exercise and recovery protocols}

The exercise commenced with a warmup of 5 min on a seated bicycle (Cybex, Minnesota, USA) at 70 revolutions per minute with zero resistance. Following the warm-up, quadriceps tightness was assessed by measuring the distance between heel and buttocks $(\mathrm{cm})$ during a maximal stretch of the muscle group without using the hand to aid the stretch. All participants were asked their pre-exercise VAS perceived pain in the quadriceps area. The 1 repetition maximum (1 RM) was ascertained to $5 \mathrm{~kg}$ accuracy by attempting 1 repetition of a knee extension using a seated knee extension machine fixed at $40 \mathrm{~kg}$ (Cybex, Minnesota, USA) and, if successful, attempt 1 repetition at increments of $5 \mathrm{~kg}$ until the exercise was not possible. The value below this failure level was taken as the $1 \mathrm{RM}$. Ideally, the $1 \mathrm{RM}$ would be found at a preceding date. However, because of time, travel and cost constraints, this was not always achievable.

The eccentric exercise protocol was based on that of Rhea et al., which produced clinical DOMS with peak pain VAS in a static stretch control group of 70/100 at $48 \mathrm{~h}$ post-exercise [9]. Eccentric exercises were performed on a seated knee extension machine consisting of negative repetition knee extensions, $4 \times 10$ repetitions at $60 \% 1 \mathrm{RM}$ unless prevented by fatigue. The exercise was standardised by timing the eccentric phase of the exercise to be completed in $6 \mathrm{~s}$, and the concentric phase performed as quickly as possible. On the final set, the participant was encouraged to continue the exercises to exhaustion.

\section{WBV protocol group}

Low-frequency vibrations $(30 \mathrm{~Hz})$ at amplitude of $4 \mathrm{~mm}$ were used. While WBV has been studied at frequencies from 12 to $50 \mathrm{~Hz}$ and amplitudes from 1 to $5 \mathrm{~mm}$, these parameters were chosen in order to facilitate comparison with the largest number of other studies, which employ the $30-35 \mathrm{~Hz}$ range $[9,30,47]$. Although the acute effects of different frequencies of WBV $(20$ and $40 \mathrm{~Hz})$ on muscle performance and flexibility have been directly compared, their impact on DOMS was not reported [24]. Each participant completed three sets of 2 min each, with 2 min rest between sets. This protocol (including rests between sets) was selected to reduce the level of fatigue from vibrations [24]. The first minute of each set was spent with $30 \mathrm{~s}$ stretching the right quadriceps (Fig. 1).

The second $30 \mathrm{~s}$ was spent stretching the left quadriceps. The second minute was spent in a squatting position with $90^{\circ}$ knee flexion with the knees over the feet and the back held in a neutral position (Fig. 2). The protocol used a Pro5 Power Plate ${ }^{\circledR}$ (Power Plate International Ltd., London, UK).

\section{Stretching (control) group}

The control group performed the stretches alongside the WBV group simultaneously, but in the absence of WBV. A quadriceps stretch was produced by the ipsilateral hand holding the foot and producing maximal knee flexion while extending the hip (Fig. 1). 


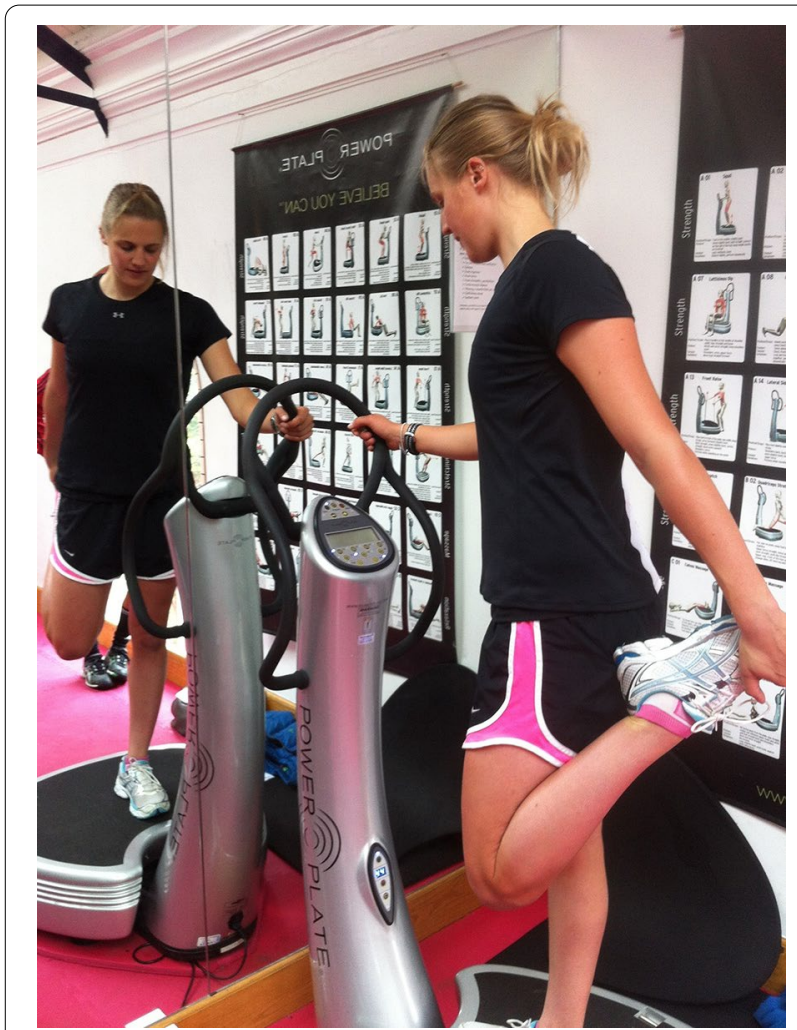

Fig. 1 Quadriceps stretch during WBV therapy

\section{Visual analogue scale and quadriceps tightness}

The participants documented their pain/soreness on a VAS ranging from 0 to 100 with 100 being maximal pain imaginable and 0 being no pain [53]. In the prone position, quadriceps tightness was measured as described above. Measurements were performed by a single investigator (JG). Anthropometry is usually very reliable, and independent reliability measurements were therefore not undertaken $[54,55]$.

\section{Statistical analysis}

Data were stored in a spreadsheet and analysed in $\mathrm{R}$ version 3.4.3 (R Core Team, Vienna, Austria). Descriptive analysis was used to compare group characteristics. The area under the curve for serial pain VAS was calculated, and distributions were assessed with histograms and box plots to confirm Normal distribution. Group means were compared using the two-sample Welch $t$ test. Serial quadriceps length measurements were expressed as proportions of the pre-exercise measurement and log-transformed before calculation of the area under the curve (to re-scale around 0). The distributions of areas under the curve were skewed, and therefore group medians were compared using the Wilcoxon rank-sum test.

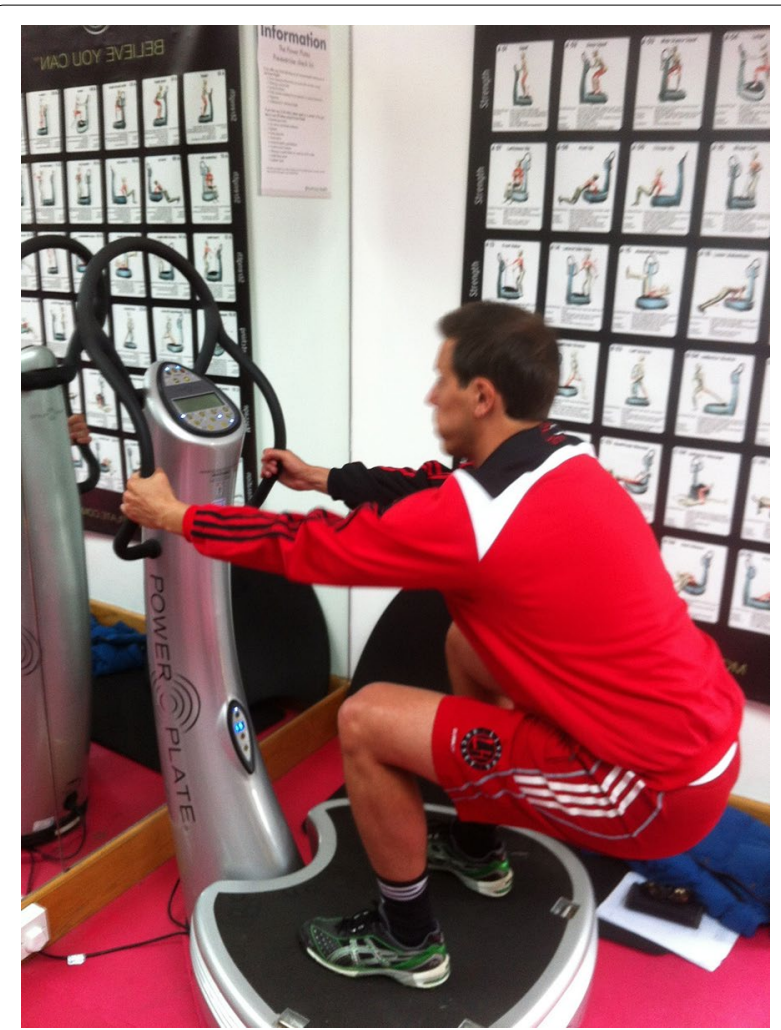

Fig. 2 Squat position during WBV therapy

\section{Results}

A total of 22 participants (14 males and 8 females) were recruited and allocated to either the WBV or stretchonly control group between June and July 2012. Eleven participants were randomised to each group. The two groups demonstrated similar characteristics at baseline (Fig. 3).

\section{Participants}

There were no statistically significant differences in age, playing ability, sex and training intensity between the groups.

Serial pain VAS for stretch-only and WBV groups was compared (Table 2; Fig. 4). Mean areas under the curve were 115 (95\% confidence interval 68-162) and 61 (95\% CI 37-85) units for stretch-only and WBV groups, respectively $(p=0.04)$.

Serial quadriceps tightness measurements were also compared (Table 2; Fig. 5). Mean areas under the (logtransformed) curve were 0.92 (95\% CI $0.05-1.78)$ and -0.11 (95\% CI -0.39 to 0.18$)$ units for stretch-only and WBV groups, respectively $(p=0.02)$.

All participants returned to normal training within 2 weeks. 


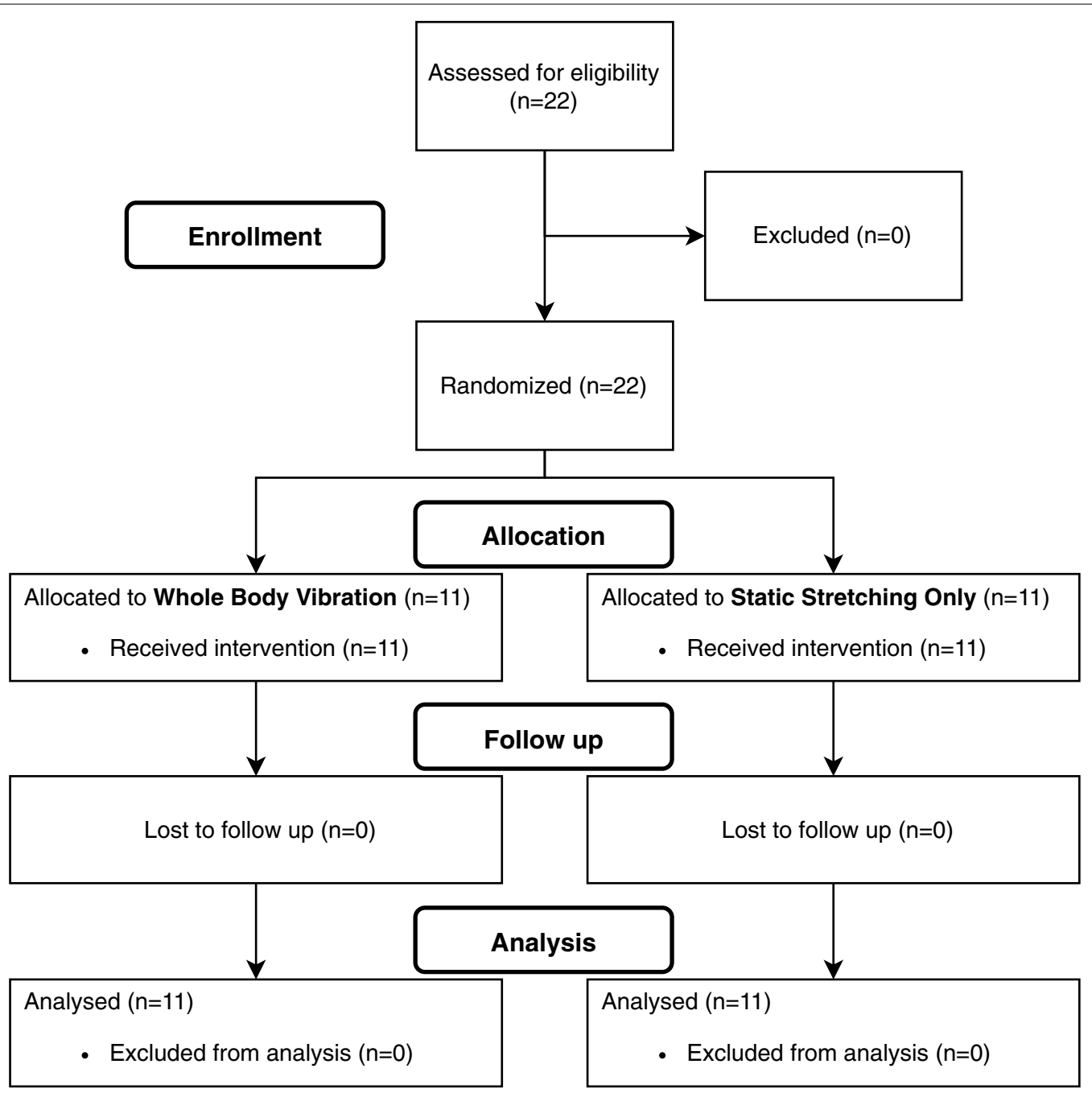

Fig. 3 CONSORT diagram

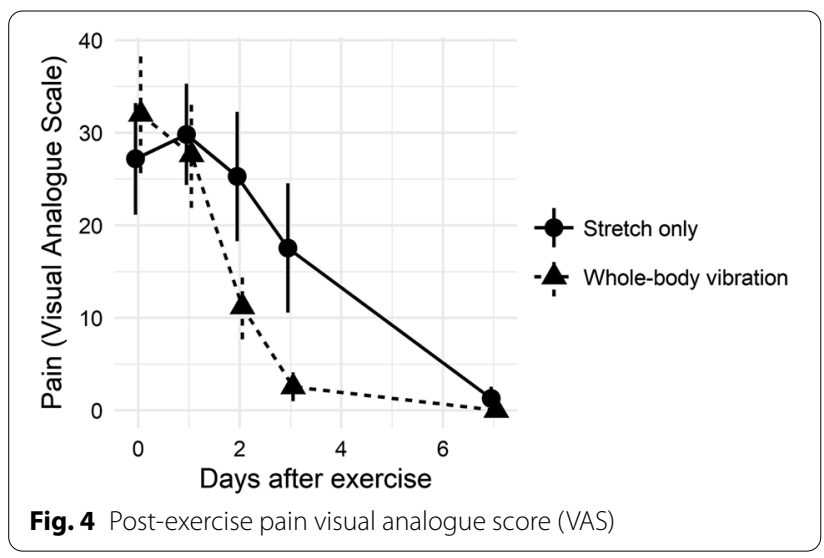

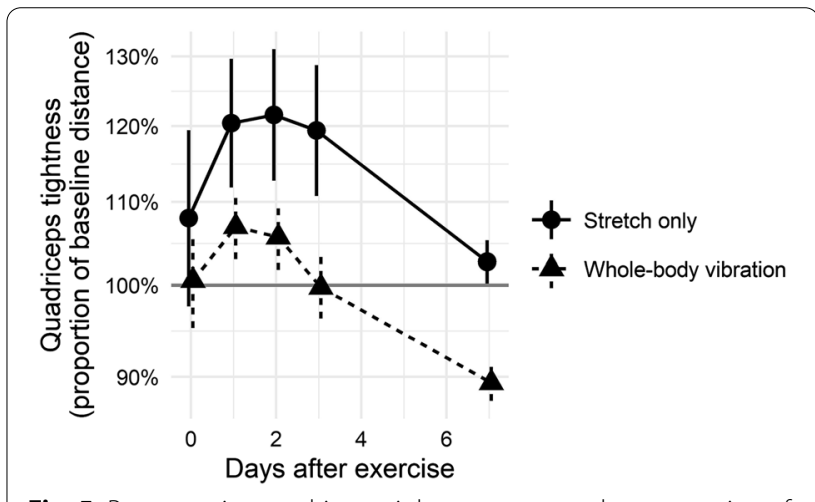

Fig. 5 Post-exercise quadriceps tightness expressed as proportion of baseline distance between heel and buttock on maximal voluntary knee flexion 


\section{Discussion}

We performed a randomised controlled trial of postexercise WBV for the reduction in DOMS in elite hockey players. Both pain and muscle tightness were significantly improved after WBV in comparison with stretching alone. The WBV therapy regime did not require additional time spent on recovery above static stretching and therefore may be integrated into existing training programmes with minimal disruption.

Results from previous studies of post-exercise WBV for prevention of DOMS have recruited either recreational or non-athletes. Results have been equivocal with no significant improvement in pain reported by five randomised trials [41-44, 46]. However, improvements in pain were found by two other trials and other literature studies $[9,39,47]$. It is established that physiological responses to exercise including the development of DOMS are altered by training, and the novel use of elite athletes in the present study may partly explain our positive findings $[50,51]$. Wide variation in treatment protocols (including timing and number of sessions, vibration frequency, etc.), control group selection, exercise protocols used to induce DOMS, and outcome measures used also underlie variation in results. For research purposes, consensus around measures of DOMS and gold standard control groups is highly desirable.

Other post-exercise studies of vibration therapy have employed local vibration rather than WBV. Results from these studies have been generally favourable [20, 37, 38, $40,48,49]$. Comparisons of local vibration with WBV have found local vibration superior for lower limb flexibility, but no difference in jump performance [56, 57]. The effects of the two modalities on DOMS have not been directly compared and should be the subject of future studies.

This trial investigated post-exercise vibration therapy. Vibration therapy has been most often studied as a recovery modality, but favourable results have been reported by multiple studies of its pre-exercise use [32, 33, 36]. In a trial which compared the use of local vibration before or after exercise with a third control group, pre-exercise therapy produced greater reductions in pain and laboratory measures of muscle damage than post-exercise therapy [35]. Further investigation is required directly comparing pre- to post-exercise vibration therapy.

The influence on results of confounding factors is minimised by the use of randomised controlled trial methodology. In addition, the selection of stretching alone for the control group provides 'real-world' relevance, because stretching remains the most common recovery modality used by both recreational and elite athletes and is widely practised to reduce muscle pain [58]. These data are a relatively novel contribution to knowledge of the effects of post-exercise WBV therapy for prevention of DOMS in elite athletes. Blinding was impractical due to the nature of the intervention, and subjective VAS results may therefore be influenced by the placebo effect. Lack of blinding could also have influenced performance in quadriceps length testing, as well as introducing assessment bias in assessors. This could be minimised in future studies comparing related vibration therapy protocols, where blinding is more feasible. Reliability analysis of quadriceps length measurement was not undertaken, and measurement error is therefore also possible. However, the reliability of anthropometry is generally high $[54,55]$. The training programme used has previously been shown to induce clinical DOMS, and therefore all participants completing the training programme were included in analysis without further confirmation or quantification of DOMS [9]. However, there is evidence that the same training program can result in variable stimuli to muscles in different individuals, and it is possible that the training programme did not produce 'true' DOMS in all participants [59]. Pain and stiffness are measures of function but are not direct evidence of the pathology of DOMS. Bias resulting from this effect is minimised by randomisation. Additionally, the delayed peak mean pain and loss of flexibility observed do suggest the presence of true DOMS. Future studies with adequate sample size may include subgroup analyses of participants with confirmed DOMS (based, for example, on laboratory testing or threshold clinical measurements). Sample size was relatively small although comparable with previous studies and necessarily limited by the availability of elite hockey players to participate [9, 20, 33, 38]. While the study was adequately powered, a greater sample size would be desirable for accurate estimation of effect sizes. It was not possible to enforce complete rest during the 7-day post-exercise observation period, and some participants may have performed limited active recovery through manual or physical occupations. Bias resulting from this is minimised by randomisation, but may have influenced results. As some variable physical activity is inevitable in 'real' settings, future studies should record such activity and consider incorporating it into the recovery protocol. The physiological differences between male and female athletes may make comparison between the two sexes inaccurate. However, most studies have demonstrated a similar level of muscle damage following eccentric exercise in both sexes [60]. Females can be more prone to muscle damage than males post-exercise $[60,61]$, but may recover quickly with an attenuated inflammatory response [60]. Due to time, travel and cost constraints, some participants were not able to attend an additional 
initial appointment to measure the 1RM, and this was done before beginning the eccentric exercise protocol. While this additional exertion may have had an impact on performance of the exercise protocol, its impact on the development of clinical DOMS or on treatment effects is not likely to be substantial.

\section{Conclusions}

WBV can be used in elite athletes to reduce the signs and symptoms of DOMS post-eccentric exercise. This finding could be extrapolated to other multi-sprint sports. Future studies could be performed with larger numbers in a randomised controlled trial with better controls to compare the effect on athletes from various sporting modalities, with the addition of muscle biopsy to further assess histological evidence of DOMS.

\section{Abbreviations}

DOMS: Delayed onset muscle soreness; WBV: Whole-body vibration; AUC: Areas under the curve; VAS: Visual analogue score; RM: Repetition maximum.

\section{Acknowledgements}

The authors would like to thank the following facilities and sports clubs: The Royal London Pathology Department and the Blizard Institute; Dr Mark Walker, Consultant Neuropathologist, Southampton NHS Foundation Trust; Fareham Hockey Club; Surbiton Hockey Club; Nuffield Health Gym, Surbiton; and Fareham Leisure Centre, Everyone Active.

\section{Authors' contributions}

HA carried out the analysis and drafted the manuscript; JG performed the testing; JG, MA, DM, FM and NM designed the study and edited the manuscript. All the authors approved the final version of the manuscript.

\section{Funding}

Open Access funding enabled and organized by Projekt DEAL. No external source of funding was used.

\section{Availability of data and materials}

Neither ethical approval nor informed consent was sought for public deposit of data, which are therefore not made publicly available.

\section{Declarations}

Ethics approval and consent to participate

Approval was obtained from the Queen Mary Research Ethics Committee (QMREC2012/50). Participants were provided written and verbal information about participation. All subjects gave informed consent to participate.

\section{Consent for publication}

Participants shown in Figs. 1 and 2 gave consent to publish their images.

\section{Competing interests}

Professor Maffulli is the Editor-in-Chief of the Journal of Orthopaedic Surgery and Research.

\section{Author details}

'Department of Trauma and Orthopaedics, Great Western Hospital, Swindon, UK. ${ }^{2}$ Centre for Sports and Exercise Medicine, William Harvey Research Institute, Queen Mary University of London, London, UK. ${ }^{3}$ Department of Orthopaedic, Trauma, and Reconstructive Surgery, RWTH Aachen University Hospital, Pauwelsstraße 30, 52074 Aachen, Germany. ${ }^{4}$ Department of Trauma and Orthopaedics, University of Salerno School of Medicine, Surgery and Dentistry, Salerno, Italy.

Received: 4 September 2021 Accepted: 30 September 2021

Published online: 12 October 2021

\section{References}

1. Hough T. Ergographic studies in muscular fatigue and soreness. J Boston Soc Med Sci. 1900;5:81-92.

2. Cheung K, Hume P, Maxwell L. Delayed onset muscle soreness : treatment strategies and performance factors. Sports Med Auckl NZ. 2003;33:145-64.

3. Armstrong RB, Warren GL, Warren JA. Mechanisms of exercise-induced muscle fibre injury. Sports Med AuckI NZ. 1991;12:184-207.

4. Pyne DB. Exercise-induced muscle damage and inflammation: a review. Aust J Sci Med Sport. 1994;26:49-58.

5. McHugh MP, Connolly DA, Eston RG, Gleim GW. Exercise-induced muscle damage and potential mechanisms for the repeated bout effect. Sports Med Auckl NZ. 1999;27:157-70.

6. Maffulli N, Oliva F, Frizziero A, Nanni G, Barazzuol M, Via AG, et al. ISMuLT guidelines for muscle injuries. Muscles Ligaments Tendons J. 2013:3:241-9.

7. Maffulli N, Del Buono A, Oliva F, Giai Via A, Frizziero A, Barazzuol M, et al. Muscle injuries: a brief guide to classification and management. Transl Med UniSa. 2015;12:14-8.

8. Silva JR, Rumpf MC, Hertzog M, Castagna C, Farooq A, Girard O, et al. Acute and residual soccer match-related fatigue: a systematic review and meta-analysis. Sports Med AuckI NZ. 2018;48:539-83.

9. Rhea MR, Bunker D, Marín PJ, Lunt K. Effect of iTonic whole-body vibration on delayed-onset muscle soreness among untrained individuals. J Strength Cond Res. 2009;23:1677-82.

10. Zainuddin Z, Newton M, Sacco P, Nosaka K. Effects of massage on delayed-onset muscle soreness, swelling, and recovery of muscle function. J Athl Train. 2005;40:174-80.

11. Sellwood KL, Brukner P, Williams D, Nicol A, Hinman R. Ice-water immersion and delayed-onset muscle soreness: a randomised controlled trial. $\mathrm{Br}$ J Sports Med. 2007:41:392-7.

12. Andersson H, Raastad T, Nilsson J, Paulsen G, Garthe I, Kadi F. Neuromuscular fatigue and recovery in elite female soccer: effects of active recovery. Med Sci Sports Exerc. 2008;40:372-80.

13. Vickers AJ, Fisher P, Smith C, Wyllie SE, Lewith GT. Homoeopathy for delayed onset muscle soreness: a randomised double blind placebo controlled trial. Br J Sports Med. 1997;31:304-7.

14. Barlas P, Robinson J, Allen J, Baxter GD. Lack of effect of acupuncture upon signs and symptoms of delayed onset muscle soreness. Clin Physiol Oxf Engl. 2000;20:449-56.

15. Bergeron-Vézina K, Corriveau H, Martel M, Harvey M-P, Léonard G. Highand low-frequency transcutaneous electrical nerve stimulation does not reduce experimental pain in elderly individuals. Pain. 2015;156:2093-9.

16. Hasson S, Mundorf R, Barnes W, Williams J, Fujii M. Effect of pulsed ultrasound versus placebo on muscle soreness perception and muscular performance. Scand J Rehabil Med. 1990;22:199-205.

17. Hasson SM, Daniels JC, Divine JG, Niebuhr BR, Richmond S, Stein PG, et al. Effect of ibuprofen use on muscle soreness, damage, and performance: a preliminary investigation. Med Sci Sports Exerc. 1993;25:9-17.

18. Jacobs SC, Bootsma AL, Willems PW, Bär PR, Wokke JH. Prednisone can protect against exercise-induced muscle damage. J Neurol. 1996:243:410-6.

19. Saxton JM, Donnelly AE, Roper HP. Indices of free-radical-mediated damage following maximum voluntary eccentric and concentric muscular work. Eur J Appl Physiol. 1994;68:189-93.

20. Broadbent S, Rousseau JJ, Thorp RM, Choate SL, Jackson FS, Rowlands DS. Vibration therapy reduces plasma IL 6 and muscle soreness after downhill running. Br J Sports Med. 2010;44:888-94.

21. Bosco C, Colli R, Introini E, Cardinale M, Tsarpela O, Madella A, et al. Adaptive responses of human skeletal muscle to vibration exposure. Clin Physiol Oxf Engl. 1999;19:183-7. 
22. Bosco C, lacovelli M, Tsarpela O, Cardinale M, Bonifazi M, Tihanyi J, et al. Hormonal responses to whole-body vibration in men. Eur J Appl Physiol. 2000:81:449-54.

23. Delecluse $C$, Roelants M, Diels R, Koninckx E, Verschueren S. Effects of whole body vibration training on muscle strength and sprint performance in sprint-trained athletes. Int J Sports Med. 2005;26:662-8.

24. Cardinale M, Lim J. The acute effects of two different whole body vibration frequencies on vertical jump performance. Med Sport (Roma). 2003;56:287-92

25. Cochrane DJ, Stannard SR. Acute whole body vibration training increases vertical jump and flexibility performance in elite female field hockey players. Br J Sports Med. 2005;39:860-5.

26. Veqar Z, Imtiyaz S. Effect of vibration in prevention of delayed onset muscle soreness: a recent update. J Physiother Sports Med. 2012;1:75-85.

27. Martin BJ, Park HS. Analysis of the tonic vibration reflex: influence of vibration variables on motor unit synchronization and fatigue. Eur J Appl Physiol. 1997;75:504-11.

28. Kerschan-Schindl K, Grampp S, Henk C, Resch H, Preisinger E, Fialka-Moser $\checkmark$, et al. Whole-body vibration exercise leads to alterations in muscle blood volume. Clin Physiol Oxf Engl. 2001;21:377-82.

29. Legleu C, Candia-Luján R, De León Fierro L, Sánchez O, Candia-Sosa K. Vibration as preventive therapy and treatment of delayed onset muscle soreness. A systematic review. Arch Med Deporte. 2016;173:194-9.

30. Lu X, Wang Y, Lu J, You Y, Zhang L, Zhu D, et al. Does vibration benefit delayed-onset muscle soreness? A meta-analysis and systematic review. J Int Med Res. 2019;47:3-18.

31. Imtiyaz S, Veqar Z, Shareef MY. To compare the effect of vibration therapy and massage in prevention of delayed onset muscle soreness (DOMS). J Clin Diagn Res JCDR. 2014;8:133-6.

32. Bakhtiary AH, Safavi-Farokhi Z, Aminian-Far A. Influence of vibration on delayed onset of muscle soreness following eccentric exercise. Br J Sports Med. 2007;41:145-8.

33. Aminian-Far A, Hadian M-R, Olyaei G, Talebian S, Bakhtiary AH. Wholebody vibration and the prevention and treatment of delayed-onset muscle soreness. J Athl Train. 2011;46:43-9

34. Kamandani R, Ghazalian F, Ebrahim K, Ghassembaglou N, Shiri Piraghaj M, Khorram A. The effect of acute vibration training on delayed onset muscle soreness in young non-athlete women. Health Scope. 2013;2:119-24.

35. Kim J-Y, Kang D-H, Lee J-H, Se M, Jeon J-K. The effects of pre-exercise vibration stimulation on the exercise-induced muscle damage. J Phys Ther Sci. 2017;29:119-22.

36. Magoffin RD, Parcell AC, Hyldahl RD, Fellingham GW, Hopkins JT, Feland JB. Whole-body vibration as a warm-up before exercise-induced muscle damage on symptoms of delayed-onset muscle soreness in trained subjects. J Strength Cond Res. 2018.

37. Koeda T, Anda T, Inoue T, Kamisaka K, Tsukamoto S, Torikawa T, et al. A trial to evaluate experimentally induced delayed onset muscle soreness and its modulation by vibration. Environ Med. 2003;47:26-30.

38. Lau WY, Nosaka K. Effect of vibration treatment on symptoms associated with eccentric exercise-induced muscle damage. Am J Phys Med Rehabil. 2011;90:648-57

39. Pinto N, Monteiro M, Arthur A, Paiva D, Meyer P, Santos-Filho S, et al. Effectiveness of a protocol involving acute whole-body vibration exercises in an adult and health individual with delayed-onset muscle soreness observed after running: a case report. J Med Med Sci. 2011;2:612-7.

40. Mohammadi $H$, Sahebazamani M. Influence of vibration on some of functional markers of delayed onset muscle soreness. Int J Appl Exerc Physiol. 2012;1.

41. Xanthos PD, Lythgo N, Gordon BA, Benson AC. The effect of whole-body vibration as a recovery technique on running kinematics and jumping performance following eccentric exercise to induce delayed-onset muscle soreness. Sports Technol. 2013;6:112-21.

42. Wheeler AA, Jacobson BH. Effect of whole-body vibration on delayed onset muscular soreness, flexibility, and power. J Strength Cond Res. 2013;27:2527-32.
43. Dabbs NC. Effects of whole body vibration on vertical jump performance following exercise induced muscle damage. Int J Kinesiol Sports Sci. 2014:2:23-30.

44. Dabbs NC, Black CD, Garner J. Whole-body vibration while squatting and delayed-onset muscle soreness in women. J Athl Train. 2015;50:1233-9.

45. Fuller JT, Thomson RL, Howe PRC, Buckley JD. Vibration therapy is no more effective than the standard practice of massage and stretching for promoting recovery from muscle damage after eccentric exercise. Clin J Sport Med. 2015;25:332-7.

46. Nepocatych S, Balilionis G, Wingo J, Bishop P. Acute effect of lower-body vibration as a recovery method after fatiguing exercise. Montenegrin J Sports Sci Med. 2015;4:11-6.

47. Timon R, Tejero J, Brazo-Sayavera J, Crespo C, Olcina G. Effects of wholebody vibration after eccentric exercise on muscle soreness and muscle strength recovery. J Phys Ther Sci. 2016;28:1781-5.

48. Cochrane DJ. Effectiveness of using wearable vibration therapy to alleviate muscle soreness. Eur J Appl Physiol. 2017;117:501-9.

49. Iodice P, Ripari P, Pezzulo G. Local high-frequency vibration therapy following eccentric exercises reduces muscle soreness perception and posture alterations in elite athletes. Eur J Appl Physiol. 2019:119:539-49.

50. Byrnes WC, Clarkson PM. Delayed onset muscle soreness and training. Clin Sports Med. 1986;5:605-14.

51. Schwane JA, Williams JS, Sloan JH. Effects of training on delayed muscle soreness and serum creatine kinase activity after running. Med Sci Sports Exerc. 1987;19:584-90.

52. Spencer M, Rechichi C, Lawrence S, Dawson B, Bishop D, Goodman C. Time-motion analysis of elite field hockey during several games in succession: a tournament scenario. J Sci Med Sport. 2005;8:382-91.

53. Bourdel N, Alves J, Pickering G, Ramilo I, Roman H, Canis M. Systematic review of endometriosis pain assessment: how to choose a scale? Hum Reprod Update. 2015;21:136-52.

54. Androutsos O, Anastasiou C, Lambrinou C-P, Mavrogianni C, Cardon G, Van Stappen $\mathrm{V}$, et al. Intra- and inter-observer reliability of anthropometric measurements and blood pressure in primary schoolchildren and adults: the Feel4Diabetes-study. BMC Endocr Disord. 2020;20:27.

55. Stomfai S, Ahrens W, Bammann K, Kovács E, Mårild S, Michels N, et al. Intra- and inter-observer reliability in anthropometric measurements in children. Int J Obes. 2005:2011(35 Suppl 1):S45-51.

56. Kurt C. Alternative to traditional stretching methods for flexibility enhancement in well-trained combat athletes: local vibration versus whole-body vibration. Biol Sport. 2015;32:225-33.

57. Özsu I, Sağiroğlu I, Kurt C, Pekünlü E. Comparing the effectiveness of whole body vibration and local vibration exercise on counter-movement jump performance and its residual characteristics in well-trained athletes. Int J Sports Exerc Train Sci. 2017;3:16-16.

58. Crowther F, Sealey R, Crowe M, Edwards A, Halson S. Team sport athletes' perceptions and use of recovery strategies: a mixed-methods survey study. BMC Sports Sci Med Rehabil. 2017;9:6.

59. McPhee JS, Williams AG, Stewart C, Baar K, Schindler JP, Aldred S, et al. The training stimulus experienced by the leg muscles during cycling in humans. Exp Physiol. 2009:94:684-94.

60. Stupka N, Lowther S, Chorneyko K, Bourgeois JM, Hogben C, Tarnopolsky MA. Gender differences in muscle inflammation after eccentric exercise. J Appl Physiol Bethesda MD. 1985;2000(89):2325-32.

61. Maclntyre DL, Reid WD, Lyster DM, McKenzie DC. Different effects of strenuous eccentric exercise on the accumulation of neutrophils in muscle in women and men. Eur J Appl Physiol. 2000;81:47-53.

\section{Publisher's Note}

Springer Nature remains neutral with regard to jurisdictional claims in published maps and institutional affiliations. 